\title{
Targeted Therapies Development in the Treatment of Advanced Nonsmall Cell Lung Cancer
}

\author{
Cesare Gridelli ${ }^{1}$ and Enriqueta Felip ${ }^{2}$ \\ ${ }^{1}$ Division of Medical Oncology, "S.G. Moscati” Hospital, 83100 Avellino, Italy \\ ${ }^{2}$ Department of Medical Oncology, Vall d'Hebron University Hospital, 8035 Barcelona, Spain
}

Correspondence should be addressed to Cesare Gridelli, cgridelli@libero.it

Received 4 October 2011; Accepted 4 October 2011

Copyright (c) 2011 C. Gridelli and E. Felip. This is an open access article distributed under the Creative Commons Attribution License, which permits unrestricted use, distribution, and reproduction in any medium, provided the original work is properly cited.

Nonsmall cell lung cancer (NSCLC) is diagnosed in the majority of patients in advanced stage of disease. In this setting, the prognosis is very poor with median survival of 912 months and palliative chemotherapy being the standard of care. However, chemotherapy in advanced disease seems to have reached a plateau with no doublet combination shown to be clinically superior to the others. Advances in the knowledge of tumor biology and mechanisms of oncogenesis has granted the singling out of several molecular targets for NSCLC treatment. Targeted therapies are designed to interfere with specific aberrant biologic pathways involved in tumorigenesis. A large amount of preclinical in vivo and in vitro data have been gathered on the antitumor properties of a number of new biological agents, both as single agents and combined with other conventional treatment modalities such as chemotherapy. Several targeted therapies have been introduced in cancer treatment, and in particular gefitinib and erlotinib-two epidermal growth factor receptor tyrosine kinase inhibitors (EGFR TKI) and bevacizumab (an antiangiogen agent) have been introduced in advanced NSCLC, and others are in current clinical development.

The present issue includes 5 papers dedicated to targeted therapies of NSCLC, 2 on basic science and 3 focusing on medical treatment. The first paper by K. S. S. Enfield et al. deals with the role of microRNA gene dosage alteration assessment in order to define potential tumor drug resistance and response to chemotherapy. The authors, by instigating a logical stepwise strategy, have identified specific microRNAs that are associated with resistance to several chemotherapeutic agents and provide a proof-of-principle demonstration of how these various databases may be exploited to derive relevant pharmacogenomic results. The second paper is a complete review on targeted therapies in NSCLC. The authors describe the identified tumor target and biomarkers opening the field of personalized medicine. They deal with already registered drugs in current clinical practice and the new agents under current investigation. R. Costanzo et al. in the third paper reviewed the role of gefitinib in the treatment of advanced NSCLC harbouring an activating EGFR mutation. Gefitinib and erlotinib can be considered the standard first-line treatment in this subgroup of patients based on their superiority showed in terms of progressionfree survival, response rate, and quality of life as compared to chemotherapy. To date gefitinib has provided data on Asians only and has not been approved in the USA while erlotinib, with data on both Asians and Caucasians, has been approved worldwide.

When added to chemotherapy, continuous treatment with tyrosine kinase inhibitors (TKIs) offers no benefit in comparison with chemotherapy alone. This may be due to the antagonistic effect of TKIs and chemotherapy: cells are pushed into the G-0 phase of the cell cycle and are therefore resistant to cytotoxic drugs. In the fourth paper M. Zwitter et al. report the results of a phase II study in patients with advanced NSCLC with EGFR mutation using an intermittent schedule of erlotinib and chemotherapy with cisplatin plus gemcitabine in order to overcome the previously described negative interference of drugs. The authors observed even on a small patients' population 
interesting results and mild toxicity. In the last, paper Escobar M. et al. make a state-of-the-art review based on the available literature regarding the use of bortezomib as a single agent or in combination with chemotherapy in patients with lung cancer. The ubiquitin-proteasome system like other cellular pathways is critical for the proliferation and survival of cancer cells; thus, proteosome inhibition has become a very attractive anticancer therapy.

Cesare Gridelli

Enriqueta Felip 


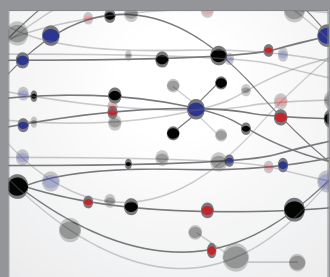

The Scientific World Journal
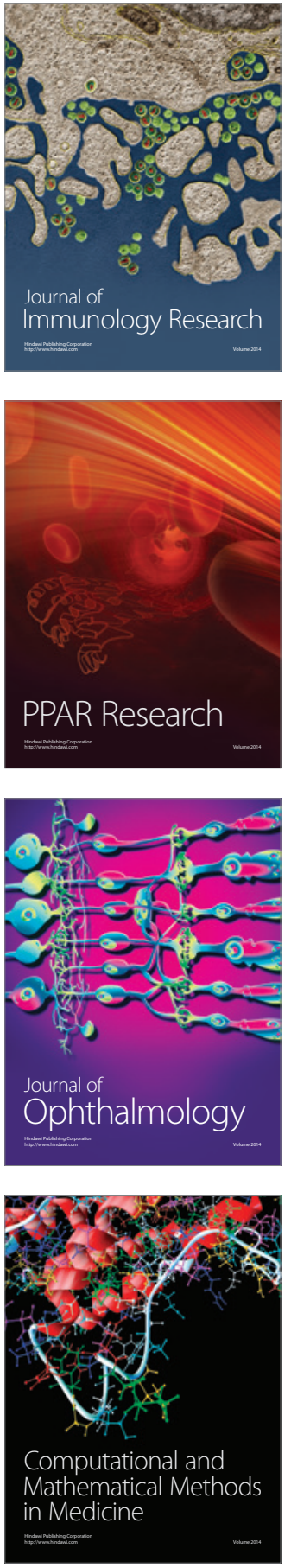

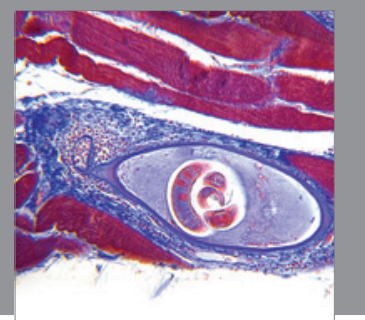

Gastroenterology

Research and Practice
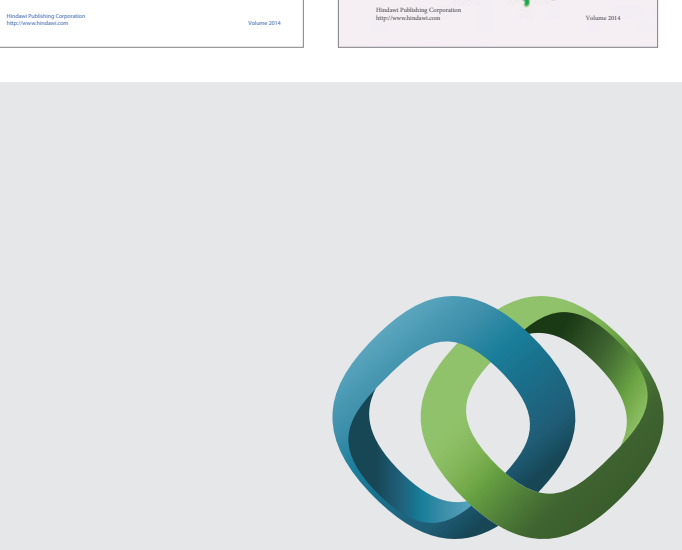

\section{Hindawi}

Submit your manuscripts at

http://www.hindawi.com
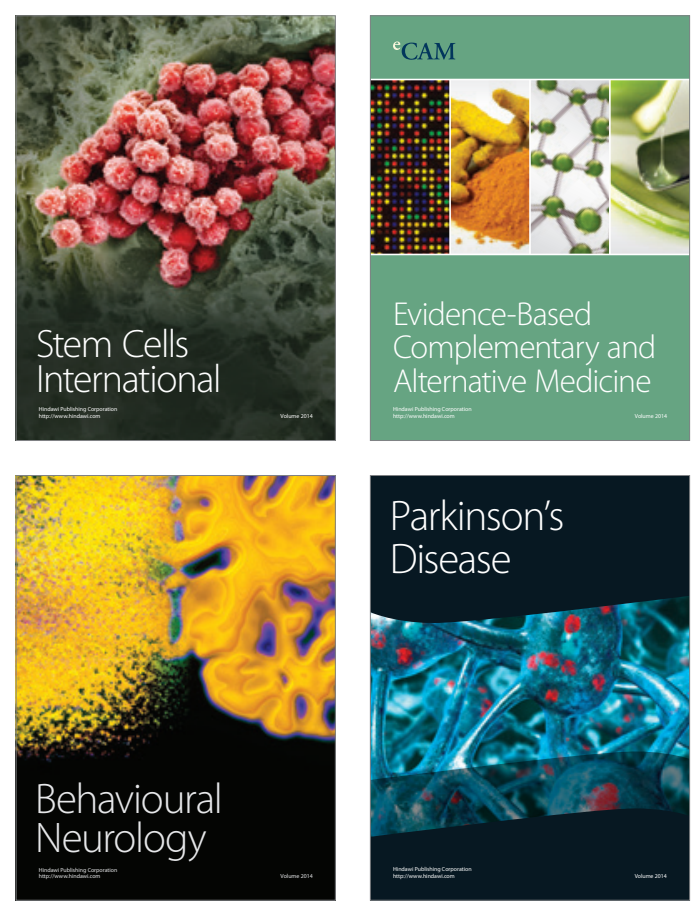

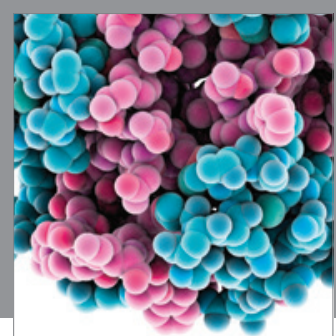

Journal of
Diabetes Research

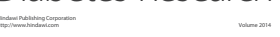

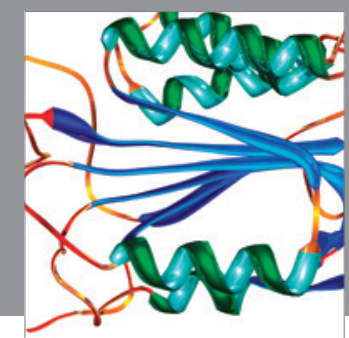

Disease Markers
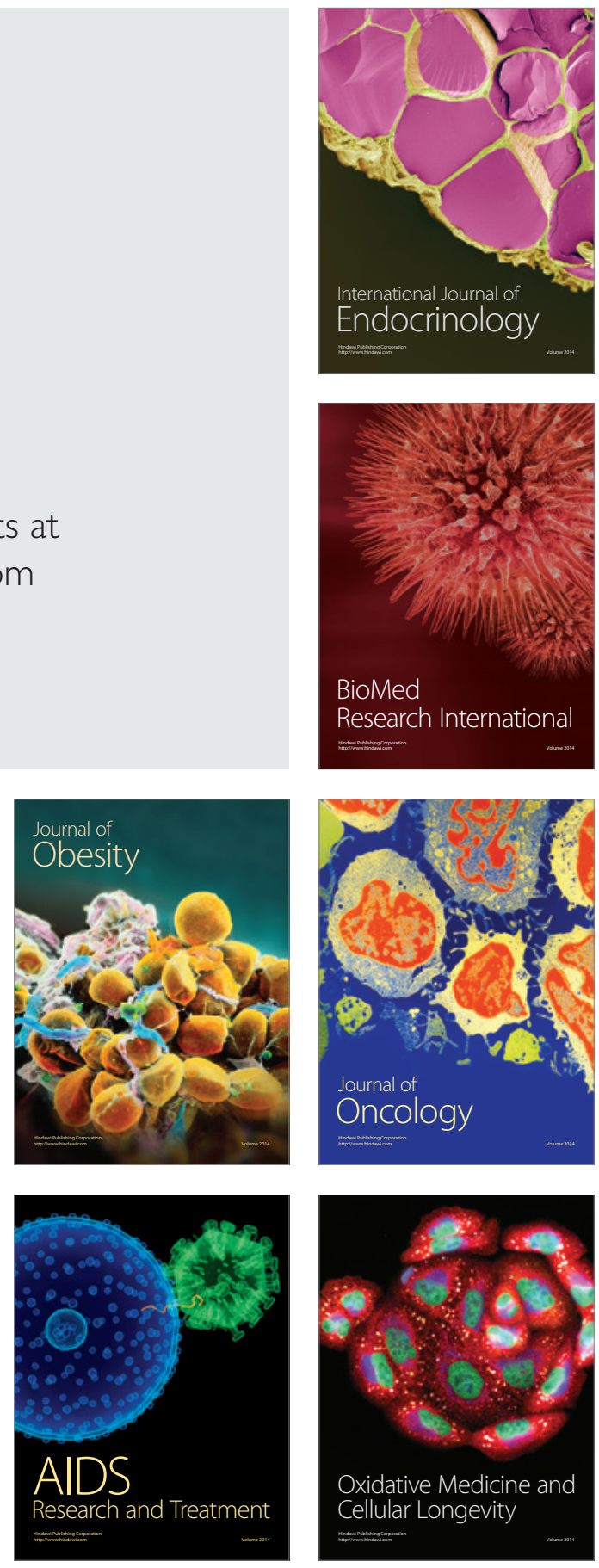